\title{
Forschendes Lehren und Lernen (FLL) in Angewandter Linguistik und Sprachdidaktik Muttersprache Deutsch: Praxiserfahrungen mit dem FLL-Beispiel Informelles Lernen in Szenen: Soziosemiotik des (Heavy) Metal
}

\author{
Teaching and Learning Research in Applied \\ Linguistics: Practical Experiences and the Practice \\ of Teaching and Learning Research based on the \\ Concrete Example Informal Learning in Scenes: \\ Socio-semiotics of (Heavy) Metal
}

\begin{abstract}
Teaching and learning research appears to be both a suitable and sustainable means to achieve an up-to-date competence for university students in applied linguistics. The understanding of and dealing with new multimodal 'glocal' semiotic systems and codes has thus emerged as a new core competence in that field. Since formal educational institutions still adhere to traditional contents, goals and methods, it has turned out to become necessary to focus on informal learning, especially when experienced in subcultures. This approach will lead to acquiring better sociosemiotic competences. The present contribution is based on data and results of imparting of knowledge about informal socio-semiotic learning at the university Wuppertal (Germany), using documents used advanced in seminars of German studies.
\end{abstract}

KEYWORDS: teaching and learning research at university, applied linguistics, informal learning, sociosemiotics, multimodality, popular music, heavy metal. 


\section{INTENTION UND STRUKTUR DES BEITRAGS}

Dieser Beitrag stellt Praxiserfahrungen mit dem hochschuldidaktischen Verfahren Forschendes Lehren und Lernen (FLL) in und für die Angewandte Linguistik vor. ${ }^{1}$ Die hier vertretene FLL-Konzeption betont die Verbindung von Lehren und Lernen, denn forschendes Lernen ist in den vorhandenen Hochschulstrukturen resp. in den zur Verfügung stehenden Formaten ohne das dieser Art Lernen angemessene Lehren nicht möglich. Der Beitrag befasst sich mit dem Lehren bei FLL-Veranstaltungen, wie es an der Bergischen Universität Wuppertal in der Fachdidaktik Deutsch realisiert wurde. Er beschreibt FLL-Prinzipien und das konkrete Vorgehen bei der kleinschrittigen Führung durch die Dozierende am FLL-Beispiel Informelles Lernen in Szenen: Soziosemiotik des (Heavy) Metal. Andere Aspekte von FLL in und für die Angewandte Linguistik und Sprachdidaktik, insbesondere die Ergebnisse der Forschungsaktivitäten der Studierenden werden in einer gesonderten Publikation zugänglich gemacht.

Die Darstellung beruht auf mehrjähriger Erfahrung (2009-2014) mit FLLVeranstaltungen. Der Beitrag konzentriert sich auf die Darstellung des von mir am intensivsten bearbeiteten FLL-Themas: Informelles Lernens in Szenen, bei dem das informelle Lernen exemplarisch an der Soziosemiotik des (Heavy) Metal festgemacht wird. Andere FLL-Themen - Jugendsprache und Sprachlicher Sexismus - werden in der o.g. Publikation vorgestellt. ${ }^{2}$

Es geht in diesem Beitrag explizit nicht um Fragen zu Vor- und Nachteilen des hochschuldidaktischen Verfahrens, nach Evaluationsmöglichkeiten, nach Lernprozessen. Leitmotiv ist vielmehr die Frage: Wie kann FLL in der Angewandten Linguistik realisiert werden? Wie ist die Balance zwischen Forschen - forschendes Lernen - und Steuerung des Forschens - forschendes Lehren - möglich? Das Attribut forschendes für Lehren ist in erster Linie eine Charakterisierung des Steuerungsprozesses; zugleich charakterisiert es aber auch die Authentizität des gemeinsamen Forschens. Welche linguistischen Fragestellungen sind zentral?

\footnotetext{
${ }^{1}$ Der Beitrag resp. die Praxiserfahrungen beziehen sich auf Germanistik-Veranstaltungen an der Bergischen Universität Wuppertal vom SS 2010 bis zum SS 2014. Die Verhältnisse in Studium und Lehre an der Bergischen Universität Wuppertal sind u.E. vergleichbar mit denjenigen anderer universitärer Hochschulen in Deutschland. Auszugehen ist bei den betreffenden FLL-Seminaren (Hauptseminare) von einer durchschnittlichen Teilnehmerzahl von $n=40$ und unterschiedlichen Leistungsnachweisen (von 2 bis $6 \mathrm{LP}$ ).

2 Ich bin Esther Galliker, Christa Stocker, Fabienne Tissot und Luise Werlen für ihr Engagement bei den ersten FLL-Veranstaltungen an der ZHAW Zürcher Hochschule für Angewandte Wissenschaften zu grossem Dank verpflichtet. Vgl Lang, Werlen (i.E.).
} 
Der Beitrag ist in zwei Teile gegliedert: Er beginnt im ersten Teil mit der Beschreibung der Konzeption Forschendes Lehren und Lernen, den Zielen des FLL, ihren strukturellen und funktionalen Merkmalen (1.1.) und ihrer semiotisch fundierten Verankerung in der Angewandten Linguistik und in der Sprachdidaktik Deutsch sowie der Diskussion des Fokus des FLL in und für die Angewandte Linguistik: die Soziosemiotik multimodaler Kommunikate (1.2.). Als Brücke zu den Praxiserfahrungen werden dann die in der Angewandten Linguistik verankerten FLL-Prinzipien (1.3.) beschrieben. Im zweiten Teil erfolgen Praxiserfahrungen in Form von Sachanalysen zum Exemplum Informelles Lernen in Szenen: Soziosemiotik des (Heavy) Metal, Informelles Lernen in Szenen (2.1.), Lernort Szene: Subkulturelles und Musikszene (2.2.), Ausgewählte Szene: (Heavy) Metal (2.3.). Szene-Lerngegenstand Multimodalität und Soziosemiotik (2.4.).

\section{KONZEPTION FORSCHENDES LEHREN UND LERNEN / FLL}

\subsection{Ziele und Merkmale des Forschenden Lehrens und Lernens}

Angewandte Linguistik ist wie jede angewandte wissenschaftliche Disziplin sowohl der Orientierung an Problemen und am Faktischen als auch der wissenschaftsdisziplinären Systematik verpflichtet. Angewandte Linguistik ist die wissenschaftliche Grundlage der Ausbildung für sprach- und kommunikationsbezogene berufliche Tätigkeiten, sei es Übersetzen, Dolmetschen, Journalismus, Management internationaler und mehrsprachiger Zusammenarbeit, Management für Aus-, Fort- und Weiterbildung oder seien es Aspekte der Medienwissenschaft und der Kommunikationswissenschaft, Entwicklungen im Wissens-, Change- und EventManagement, im sog. cultural brokerage und dergl. mehr. Sprachdidaktik der Muttersprache Deutsch ist als empirisch fundierte Fachdidaktik ein Gebiet der Angewandten Linguistik.

FLL darf als ein hochschuldidaktisches Verfahren gelten, das Professionalität und Employabilität (Berufsfähigkeit) der Studierenden ausbauen kann. ${ }^{3}$ FLL verfolgt zum einen kognitiv-emanzipatorische Ziele, wie Lernerfahrung „Das Lernen lernen”, Problemlösungsverfahren und kritische Ref-

\footnotetext{
${ }^{3}$ Der Beitrag befasst sich explizit mit FLL als hochschuldidaktisches Verfahren, wie es z.B. von Huber, Hellmer, Schneider 2013 dargestellt wird. Es geht nicht um die traditionsreichen und intensiv diskutierten Ansätze und Verfahren der Fort- und Weiterbildung von amtierenden Lehrkräften.
} 
lexion der eigenen Konzepte entwickeln, und zum andern stofflichinhaltliche Ziele, wie Erweiterung der Sachkompetenz, Aneignen von Fakten, von Wissen, Antworten auf praxisbezogene - auch v.a. sprachpolitische Fragen. FLL soll also vor allem einen Beitrag dazu leisten, einzusehen, dass Standorte - die eigenen und die Dritter - unablässig überdacht und modifiziert werden müssen und dass Strategien für die Überprüfung von Interpretationen gefunden werden müssen. Der Besuch von FLL-Veranstaltungen bietet Studierenden Einblick in Forschung, lässt Studierende eigene kleine Forschungsprojekte durchführen, verlangt von Studierenden eigene Ideen und Fragestellungen, kontrolliertes Vorgehen, Überprüfung der Vorgehensweisen und der Ergebnisse.

\subsection{Semiotisch fundierte Verankerung des FLL in der Angewandten Linguistik und in der Sprachdidaktik Muttersprache Deutsch}

Im Hinblick auf Kompetenzen in der Angewandten Linguistik resp. in der Fachdidaktik Sprache/Muttersprache Deutsch liegt ein zentraler Mehrwert des FLL im Aufbau eines reflektierten, selbstkritischen disziplinären linguistischen Wissens sowie einem Ausbau der Transfer- und Strukturierungskompetenz. Forschungswissen soll dazu beitragen, Alltagswissen transparent und veränderbar machen. Die eigene Betroffenheit soll einer Distanz zu Überzeugungen weichen und Möglichkeiten suchen, diese Überzeugungen zu hinterfragen. Gerade in "Sprachsachen" ist eine Wissenschaftsorientierung notwendig, denn die subjektive Gewissheit, Bescheid zu wissen, ist wie z.B. Bildungsdiskurse zeigen - weit verbreitet: Alle sprechen, also sind alles Fachleute für Sprachfragen.

Die Gesellschaft für Angewandte Linguistik e.V. GAL (gegründet 1968) hält fest4: "Angewandte Linguistik vermittelt zwischen Theorie und Praxis. Sie arbeitet interdisziplinär an der Lösung aller Fragestellungen und Probleme, an denen Sprache beteiligt ist - in Gesellschaft, Politik und Wirtschaft. (...) Die Angewandte Linguistik ist eine der großen Arbeitsrichtungen innerhalb der Linguistik (Sprachwissenschaft). Sie untersucht das sprachlich-kommunikative Handeln in allen Feldern der gesellschaftlichen Praxis, und zwar unter dem Aspekt der Anwendung ihrer Ergebnisse in dieser Praxis (zum Beispiel durch Beratung und Training). (...) Die Angewandte Linguistik verwendet für ihre Untersuchungen auch allgemein-linguistische Methoden

4 <http:// www.gal-ev.de/index.php/angewandte-linguistik-start> [zuletzt besucht: 16.10.2014]. 
und Erkenntnisse. Damit jedoch die Praxis Nutzen aus den linguistischen Analysen ziehen kann, sind eine angepasste Methodologie und ein Forschungsdesign notwendig, das von praktischen Problemen ausgeht und auf Problemlösungen zielt. Die Angewandte Linguistik entwickelt deshalb ihre eigenen Analysemethoden weiter, sie entwirft Konzepte für den Transfer wissenschaftlicher Erkenntnisse in die gesellschaftliche Praxis und sie untersucht Methoden der effizienten Vermittlung sprachlichen und kommunikativen Wissens (Beratung, Training, Coaching). Dabei sucht die Angewandte Linguistik die Kooperation mit der Praxis und mit anderen wissenschaftlichen Disziplinen."

Für die Sprachdidaktik Muttersprache Deutsch ist FLL besonders interessant, weil FLL im Hinblick auf kompetenzorientiertes Unterrichten ein geeignetes hochschuldidaktisches Verfahren darstellt, um Studierenden Fähigkeiten für Flexibilität und Adressatenaffinität bei der Handhabung von Unterrichtsstoffen und Unterrichtsverfahren zu vermitteln. ${ }^{5}$

Da das zentrale Thema der Angewandten Linguistik das "sprachlichkommunikative Handeln in allen Feldern der gesellschaftlichen Praxis" (s.o.) ist, steht die Auseinandersetzung mit der Spezifik des Sprachlich-Kommunikativen im Zentrum des Forschenden Lehrens und Lernens in und für die Angewandte Linguistik und Sprachdidaktik: Zeichenhaftigkeit und Zeichenprozesse (Semiosen)6 bzw. "Texte in gefassten kulturellen Kontexten"7 (Lotman 1981: 9). Dahinter steht ein semiotisches Modell von Kultur, das die Grundlage für die Untersuchung von informellem Lernen in Szenen und dessen prototypische Erscheinungsweise Soziosemiotik ermöglicht. „Kultur - das ist die Gesamtheit aller nicht vererbten Information zusammen mit den Verfahren ihrer Organisation und Speicherung. (...) Die Information ist kein fakultatives Merkmal, sondern sie ist eine der Grundvoraussetzungen menschlicher Existenz. (...) Die Auffassung, dass Kultur wesentlich Informa-

\footnotetext{
${ }^{5}$ Argumente für FLL kommen vor allem aus dem Erreichen des Kompetenzprofils, das AbsolventInnen von Lehramtsstudiengängen aufweisen müssen, und dem Erfüllen von Standards, denen die LehrerInnenAusbildung genügen muss: Lehrkräfte sind Fachpersonen für bestimmte wissenschaftliche Disziplinen sowie trans- und interdisziplinäres Arbeiten, Vermittlung von Erkenntnissen und Wege der Erkenntnisgewinnung bestimmter wissenschaftlicher Disziplinen, Wissensmanagement bei Jugendlichen, Schulorganisation und Kooperationen, v.a. auch Elternarbeit.

${ }^{6}$ Wir beziehen uns beim Konzept Semiose zum einen auf Posners Darstellung Kultursemiotik (Posner 2008), zum andern auf die semiotische Modellierung von Morris (1977; 1979).

7 Auf die Entwicklung und Verwendung des Konzepts Text gehen wir hier nicht ein. Lotmans prägnante Formulierung "statt Orientierung auf einzelne immanent abgeschlossene Texte Hinwendung zu weiter gefassten kulturellen Kontexten" (Lotman 1981: 9) muss hier genügen. In sprachdidaktischen Kontexten ist oft von einem „erweiterten Text- und Sprachbegriff" die Rede.
} 
tion ist, erklärt auch, weshalb sowohl die Träger einer jeweiligen Kultur wie auch ihre Gegner an dieser Frage so leidenschaftlich interessiert sind und ihre Konflikte die Menschheitsgeschichte ausfüllen" (Lotman 1981: 26).

Wir richten den Fokus unserer FLL-Arbeit auf das informelle Lernen in einer Musikszene. Das „unsichtbare Bildungsprogramm“ (Hitzler, Pfadenhauer 2005) der Szene - das informelle Lernen - manifestiert sich da in der Soziosemiotik multimodaler Kommunikate. Verschiedene wissenschaftliche Referenzen sind für die Umsetzung dieser Fokussierung grundlegend: In erster Linie der soziolinguistische Ansatz in Verbindung mit der Kultursemiotik, wie sie von Posner (2008) beschrieben wird und - vor allem im Hinblick auf die empirische Arbeit - die Ethnographie der Kommunikation, wie sie Hymes und Gumperz entwickelt haben.

Die zentrale Fragestellung für FLL ist dann die nach dem Aushandeln von Bedeutungen: Was bedeutet was für wen unter welchen Bedingungen mit welcher Verbindlichkeit und welcher Dauer? Wir haben es damit mit einem genuin soziolinguistischen Ansatz zu tun. ${ }^{8}$ Wir sprechen daher auch von Soziosemiotik und nicht von Sozialsemiotik. Der Begriff Soziosemiotik verweist in unserem FLL-Zusammenhang auf zwei Dimensionen: Zum einen auf eine wissenschaftliche Disziplin (vgl. insbes. Alkemeyer 2003) und zum andern auf die Zeichensysteme, die als Untersuchungsgegenstand fokussiert werden. Das Zusammenspiel dieser Codes bei den multimodalen Kommunikaten bezeichnen wir ebenfalls als Soziosemiotik.

Wir bevorzugen den in den Medienwissenschaften (vgl. Stöckl 2004) eingebürgerten Begriff Kommunikat gegenüber dem Begriff Text, wie er in der Kultursemiotik üblich ist, weil zum einen der Terminus Text im Dialog mit Studierenden permanent zu Missverständnissen führt und weil zum andern der Terminus Kommunikat den dynamisch-interaktionalen Charakter des fokussierten Lerngegenstands besser begreifen lässt. Kommunikate sind sui generis multimodal. Sie bestehen aus einer je spezifisch gestalteten Konfiguration von Codes. Die „klassische” multimodale nicht-mündliche Konfiguration, der sich die Linguistik vornehmlich widmet, ist die Text-BildKonfiguration, im Mündlichen gilt die Aufmerksamkeit der Vernetzung von verbalen und non-verbalen Zeichensystemen resp. Codes. ${ }^{9}$ Wir verwenden den Begriff Code für umreißbare semiotische Teilsysteme. ${ }^{10}$

\footnotetext{
${ }^{8}$ Fishman lässt grüßen: vgl. Fishman 1975.

${ }_{9}^{9}$ Die aktuelle Forschungsrichtung wird vertreten von Spitzmüller (2013). Die typografische Gestaltung von Bandlogos, Booklets und Homepages von Musikszenen bietet vielfältige Gelegenheiten, dieses linguistische (sic!) Feld zu untersuchen. Metal hat hier sehr viel zu bieten.

${ }^{10}$ Obwohl er an das kybernetische Sender-Empfänger-Modell erinnert, in dem Codes statischer Natur sind und nicht ausgehandelt werden. Vgl. Posner (2008: 53): „Da Systeme von
} 
Das Spannende und Aufschlussreiche am soziosemiotischen Zugriff auf multimodale Kommunikate ist die Konzentration auf die Interaktion bzw. Interdependenz der Codes. Es geht dabei nicht nur um ein Zusammenspiel der Codes, sondern darum, dass einzelne Codes und die Konfiguration der Codes bei unterschiedlichen Gruppen unterschiedliche Deutungen und Kohärenzen hervorrufen. Das scheint auf den ersten Blick sehr trivial zu sein, ist aber auf den zweiten Blick der Schlüssel zum Verstehen von Semiosen - synchron und diachron. Dieser Ansatz erweist sich für Zugänge zum informellen Lernen als besonders geeignet, und er lässt es zu, die „Produkte”, „Produktionen” und „Rezeptionen” in popularen Kulturen empirisch und konzeptuell angemessen wahrzunehmen.

\subsection{Prinzipien des Forschenden Lehrens und Lernens}

\subsubsection{Sieben FLL-Prinzipien}

I „Gesteuerte Selbstständigkeit”: Die FLL-Prinzipien heben auf die Balance von Seminarsteuerung mit ihrem kleinschrittigen Vorgehen und den selbstständigen, selbstorganisierten Forschungen der Studierenden ab.

II „Explizitheit des Ziels der FLL-Veranstaltung”: Die Zielsetzung der FLL-Veranstaltung und ihr Modell des Spiralcurriculums werden explizit verabredet.

III „Iteratives Vorgehen”: Das schrittweise resp. kleinschrittige Vorgehen wird auf organisatorischer, sachinformations- und methodenvermittelnder Ebene realisiert.

IV „Spiralmodell”: Das Informationsmanagement in Bezug auf die FLL-Thematik und die wissenschaftsorientierte Verfahrensweise der Studierenden ist spiralförmig angelegt. ${ }^{11}$ Das Überschneiden der Lernschritte ermög-

Signifikant-Signifikat-Zuordnungen als «Codes» bezeichnet werden, führt diese Überlegung [die zum semiotischen Status von Mentefakten; el] zu dem Resultat, dass sich jede Mentalität als Menge von Codes auffassen lässt."

${ }^{11}$ Wir orientieren uns hier u.a. an Aebli $(1980 ; 1981)$ und Steiner (2007) Für intensive Diskussionen dieser Literatur danke ich Simon Tiefenbach. 
licht kontinuierliche Reflexion und Anwendung des Erlernten, so dass aus Informationen Wissen aufgebaut werden kann. Zieltransparenz/Zielkonsens - Informationen - Reflexion der Informationen - Anwenden/ Transfer/Üben mit den Informationen - Fragestellungen - Erweitern der Informationen/Aufbau von Wissen - Anwenden im Diskurs/Überprüfen der Anwendung - gezielter Ausbau des Wissens - Reflektieren und Zusammenfassen - Präsentieren/Leistungskontrolle.

V „Fallstudien”: FLL setzt am sinnvollsten eine Methodologie - eine Theorie der Methode - um, die man "qualitatives Vorgehen" nennt und die auf Fallstudien und deren Vergleich beruht. Man geht von Einzelfällen aus und versucht, Erkenntnisse durch die Untersuchung von einzelnen, exemplarischen Fällen zu gewinnen (qualitativ-induktives Vorgehen). Im Vordergrund steht die Möglichkeit, ein Phänomen erkennen und verstehen zu können (Betonung des heuristischen Potentials an Stelle einer Hypothesenprüfung), und wichtiger als repräsentative Aussagen treffen zu können, ist die Auseinandersetzung mit einem „echten”, alltagsnahen, authentischem Problem (Betonung der Authentizität an Stelle der Repräsentativität).

VI „Präsentation”: Die Ergebnisse der Gruppenarbeit können unterschiedliche Schwerpunkte haben. Wenn es die Infrastrukturen der Hochschule zulassen, ist eine Ausstellung für die Hochschulöffentlichkeit ein sinnvoller Abschluss einer FLL-Veranstaltung. Auch die übliche Form der Präsentation ist angebracht, allerdings wirft dieses Vorgehen erhebliche Zeitprobleme auf, so dass einerseits das Herstellen von Posters für das Plenum der Veranstaltung, andererseits eine knappe Zusammenfassung pro Gruppe und die Zurverfügungstellung der Ergebnisse im pdf-Format. Entscheidend ist, dass alle teilnehmenden Studierenden von allen Gruppen die Ergebnisse erhalten.

VII „Rahmendes Forschungsprojekt”: Rahmendes Forschungsprojekt für die Umsetzung von FLL ist das Forschungsprojekt Soziosemiotik des europäischen Heavy Metal, das aktuell in einer europäischen Kooperation von ForscherInnen aus den Ländern Deutschland, Finnland, Großbritannien, Luxembourg, Polen und Schweiz die Thematik Analysis of the European Metal scene as a paradigm of glocality, transnational mentality and social cohesion untersucht. 


\subsubsection{Konstituenten und Ablauf des FLL-Spiralcurriculums in der FLL-Veranstaltung Informelles Lernen in Szenen: Soziosemiotik des (Heavy) Metal}

(1) Informationen an Studierende über die FLL-Spezifik und FLL-Anforderungen

Studierende, die an FLL-Seminaren teilnehmen, müssen sich für ihre Teilnahme auf der Basis von Informationen über die FLL-Spezifik und FLL-Anforderungen entscheiden und bereit sein, einen angemessenen Beitrag zur Zielerreichung der Seminars zu leisten ${ }^{12}$.

1. Erklärungen zum Forschenden Lehren und Lernen

1.1. Kommentar zu FLL-Seminaren

1.2. Strukturierung der FLL-Tätigkeit

1.3. Anleitungen zum Arbeitsprozess (nicht abgebildet)

(2) Informationen über die linguistisch-kultursemiotische Thematik und Einbindung in Kultur-, Kommunikations- und Bildungsdiskurse

Die kleineren Forschungsprojekte der Studierenden - in der Regel Fallstudien - sind in einem Forschungsprojekt mit expliziten Erkenntnisinteressen verortet. Der dadurch vermittelte, die Einzelfälle übergreifende Diskurs macht die Vorgabe von Erkenntnisinteressen und Leitkonzepten plausibel.

2. Orientierungen zum Seminarthema und zu Leitkonzepten

2.1. Seminarziel, Leitkonzepte Soziosemiotik und Informelles Lernen, Beschreibung der Musikszene, Parameter der Beschreibung (mit Beispielen von Projektmitarbeiter Stephan Knapp)

2.2. Einführung in die Thematik: Protokoll der Rezeption des Films von Sam Dunns A Headbangers Journey (Fragebogen von Projektmitarbeiterin Luise Werlen)

2.3. Einführung Rezeption Musik von Seminarteilnehmer Jan Kristian Brühne (ppt-Präsentation Zur Funktion von Text-Musik-Komplexen in religiösen Überzeugungssystemen)

2.4. Nähere Bestimmung der Musikszene (Heavy) Metal/Vorschläge für Bands (Projektmitarbeiter Florian Piwek)

(3) Organisatorisch-inhaltliche Steuerung: Bildung von Arbeitsgruppen und Vernetzung der Studierenden untereinander parallel zum Aufzeigen von Beschreibungsmöglichkeiten und inhaltlich ausgerichteten Präsentationen der Seminarleitung

\footnotetext{
12 Die Dokumente in ihrem Wortlaut werden in Lang, Werlen (i.E.) publiziert.
} 
Die Studierenden vernetzen sich untereinander und bilden nach inhaltlichen Vorgaben Arbeitsgruppen. Die inhaltlichen Aufgaben der Arbeitsgruppen werden in mehreren Arbeitsschritten erläutert und geklärt. Im Zuge der inhaltlichen Konkretisierung organisieren die Studierenden ihre Gruppenarbeit.

3. Organisatorisch-inhaltliche Steuerung der FLL-Veranstaltung

3.1. Präsentationen der Seminarleitung

3.2. Konkretisierung der Arbeitsgruppen I-VIII auf der Basis von Parametern und von Indikatoren (Projektmitarbeitende Luise Werlen, Florian Piwek, Dominik Jablonowski)

(4) Gewählte Parameter und ihre Indikatoren werden in spiralförmig angelegten Arbeitsphasen erfahrbar gemacht.

Damit konkretisieren sich die Fragestellungen der Arbeitsgruppen, die interne Arbeitsteilung und - planung sowie die Ergebnisorientierung. Der Dreischritt hier ist: Frage - Wege zur Beantwortung der Frage - Antwort auf die Frage.

4. Arbeitsphasen

4.1. Konkretisierung der Arbeitsgruppen I-VIII auf der Basis von Parametern und von Indikatoren

4.2. Übung zum Verständnis der Parameter und Indikatoren: Rezeption Iron Maiden-Dokumentation (DVD-Präsentation Dominik Jablonowski)

4.3. Leitkonzept Bricolage und Anwendungsübung im Plenum: Rezeption von Eluveitie und Dunns Global Metal mithilfe des Konzepts Bricolage

4.4. Anwendungsübung zur Beschreibung und zum Kennenlernen der eingesetzten Parameter/Rezeption Band Kreator

(5) Leistungskontrolle und Ergebnispräsentation

5. Möglichkeiten der Ergebnissicherung/-kontrolle

5.1. Abschlussfragebogen / WS 2013/2014

5.2. Pressetext zur Ausstellung Wo die wilden Kerle wohnen - Heavy Metal im Bergischen Land 26.01.2011

\subsubsection{Seminarplan}

\begin{tabular}{|c|c|l|}
\hline Sitzung & \multicolumn{1}{|c|}{ Phase } & \multicolumn{1}{c|}{ Themen der Sitzung } \\
\hline 1 & & $\begin{array}{l}\text { Zielvereinbarung; Thematik des FLL-Seminars Infor- } \\
\text { melles Lernen in Szenen: Soziosemiotik des (Heavy) Metal; } \\
\text { Vorgehen im FLL-Seminar }\end{array}$ \\
\hline
\end{tabular}




\begin{tabular}{|c|c|c|}
\hline Sitzung & Phase & Themen der Sitzung \\
\hline 2 & \multirow[t]{2}{*}{$\begin{array}{l}\text { tionen }>\text { Bildung } \\
\text { Arbeitsgruppen }\end{array}$} & $\begin{array}{l}\text { Präsentation der Seminarleitung 01: Soziolinguisti- } \\
\text { sche und kultursemiotische Verortung; Hinführung } \\
\text { zum Aspekt Szene und Soziosemiotik: Rezeption von } \\
\text { Sam Dunn's Dokumentation A Headbanger's Journey + } \\
\text { Protokoll zur Rezeption }\end{array}$ \\
\hline 3 & & $\begin{array}{l}\text { Einbetten der Thematik in Kultur-, Kommunikations- } \\
\text { und Bildungsdiskurse + Positionierung des übergrei- } \\
\text { fenden Forschungsprojekts; Diskussion Rezeption } \\
\text { von Sam Dunn's Dokumentation A Headbanger's } \\
\text { Journey }\end{array}$ \\
\hline 4 & \multirow[t]{3}{*}{$\begin{array}{l}\text { Informationen zur } \\
\text { Konzeption und zu } \\
\text { Leitkonzepten }\end{array}$} & $\begin{array}{l}\text { Diskussion der Reaktionen auf "Metal-Kontakt": } \\
\text { Frage nach Beschreibungsperspektive und - } \\
\text { möglichkeiten > Vorgabe der Parameter, Grundlage } \\
\text { Bildung von Arbeitsgruppen; Basiskonzepte: Szene, } \\
\text { informelles Lernen, Soziosemiotik, multimodale } \\
\text { Kommunikate; Präsentation der Seminarleitung } 02\end{array}$ \\
\hline 5 & & $\begin{array}{l}\text { Präsentation der Seminarleitung } 02 \text { "Bildungspro- } \\
\text { gramme”, Soziosemiotik, Multimodalität; Bildung } \\
\text { der Arbeitsgruppen; Auswahl /Vorstellen der Bands }\end{array}$ \\
\hline 6 & & $\begin{array}{l}\text { Präsentation der Seminarleitung 03; Anwendung der } \\
\text { Parameter: Rezeption Auszüge von Sam Dunn's } \\
\text { Dokumentation Global Metal }\end{array}$ \\
\hline 7 & \multirow{3}{*}{$\begin{array}{l}\text { Anwendungs- } \\
\text { übungen Parame- } \\
\text { ter//Konkreti - } \\
\text { sierung Frage- } \\
\text { stellung und Vorge- } \\
\text { hensweise }\end{array}$} & $\begin{array}{l}\text { Anwendung und Überprüfung der Parameter: Re- } \\
\text { zeption der Dokumentationen Iron Maiden und } \\
\text { Eluveitie; Vergleiche und Auswahl von ergiebigen } \\
\text { Indikatoren }\end{array}$ \\
\hline 8 & & $\begin{array}{l}\text { Transfer der Vergleichsprozesse auf Dokumentation } \\
\text { Kreator + Protokoll/Fragebogen; } \\
\text { Präsentation der Seminarleitung } 03\end{array}$ \\
\hline 9 & & $\begin{array}{l}\text { Spezifizierungen der multimodalen Konfigurationen } \\
\text { bei Iron Maiden, Kreator, Eluveitie; } \\
\text { Definitive Aufgabenteilung in den Arbeitsgruppen }\end{array}$ \\
\hline 10 & \multirow{2}{*}{$\begin{array}{l}\text { Selbständiges } \\
\text { Arbeiten }\end{array}$} & \multirow[t]{2}{*}{ Fallstudien der Arbeitsgruppen } \\
\hline$\frac{11}{12}$ & & \\
\hline 13 & \multirow{2}{*}{$\begin{array}{l}\text { Leistungs- } \\
\text { Kontrolle / Evaluie- } \\
\text { rung }\end{array}$} & \multirow{2}{*}{$\begin{array}{l}\text { [Ausstellung/Poster] Präsentationen [Durchführen } \\
\text { einer Ausstellung mit Postern und Präsentationen ist } \\
\text { Ergebnisdarstellung aller Teilnehmenden. Abschluss- } \\
\text { fragebogen. }\end{array}$} \\
\hline 14 & & \\
\hline
\end{tabular}




\section{SACHANALYSEN ZUM FLL-EXEMPLUM INFORMELLES LERNENS IN SZENEN: SOZIOSEMIOTIK DES (HEAVY) METAL}

\subsection{Informelles Lernen in Szenen}

Für Jugendliche stellen Sozialisation und Enkulturation durch Gleichaltrige existentielle Lernprozesse dar, und wesentliche Bereiche des Alltags resp. der Freizeit spielen sich in Szenen oder Cliquen ab. Soziologische und pädagogische Jugendforschung sehen Szenen bzw. Freizeitkulturen als neue Sozialisations- und Enkulturationsinstanz. ${ }^{13}$ Sie wird als Folge der Entwicklung westlicher Gesellschaften zu Gesellschaften der Individuen ${ }^{14}$ nach dem II. Weltkrieg betrachtet. Die neue Lebensphase Jugend ist auch der Entstehung von Freizeitkulturen geschuldet (u.a. Hurrelmann, Quenzel 2012). Die „Lebensphase Jungend"15 entspricht einer gesellschaftlichen Gruppierung von Menschen im Alter von 12 bis 25 Jahren, die sich natürlich im Hinblick auf soziale, politische, ökonomische, kulturelle etc Dimensionen intern stark unterscheidet.

Wir haben uns für das jugendkulturelle Konzept Szene entschlossen und nicht für das Konzept Sub- oder Gegenkultur, das in der Popkulturforschung und in der Popmusikforschung eine zentrale Rolle spielt. Der Begriff scheint uns für unsere Erkenntnisinteressen trotz seiner in weiterem und engerem Sinn verwendeten Bedeutung ${ }^{16}$ zu stark auf die Reichweite von Mentefakten bezogen: Der Begriff Szene lässt diese Reichweite offen und überlässt es der Empirie diese jeweils festzustellen. Der Begriff Subkultur impliziert eine starke Kohäsion zwischen der Symbolwelt einer Gruppe und den Alltagspraxen ihrer Mitglieder sowie die mentalitäre Trennung von der umfassenderen sozialen Welt, und er zielt eher auf die sozialen Gruppen der Popkultur als auf ihre Kommunikate.

In Kindheit und Jugend werden grundlegende Kompetenzen erworben. Entgegen traditioneller Ansichten, mit dem Abschluss der Jugend sei der Abschluss der Ausbildung verbunden, sehen Arbeitsmarkt und Politik das sog. lebenslange Lernen als gegeben bzw. als notwendig an. Es ist unmittel-

13 Vgl. Hurrelmann (2004); Hurrelmann, Quenzel (2012); Ferchhoff (1999; 2010), Baacke $(1968 ; 1972 ; 1976 ; 1987)$.

${ }^{14}$ Auch Hitzler, Niederbacher (2010: 9) stellen ihre Szeneforschung in diesen Modernisierungsdiskurs: "Zu einem allgemeinen biografischem Muster für den Heranwachsenden schlechthin (...) wurde sie allerdings tatsächlich erst im erst im 20. Jahrhundert - und in ihrer uns zwischenzeitlich geläufigen Ausprägung eigentlich erst im Kontext wirtschaftlicher Prosperität der Zeit nach dem Zweiten Weltkrieg".

${ }^{15}$ Vgl. Wicke, Ziegenrücker, Ziegenrücker (2007); Diederichsen (2014).

16 Vgl. Hügel 2003: 66-73. 
bar einleuchtend, dass man hier ausdiskutieren muss, was in diesem Zusammenhang als „Lernen” angesehen wird, denn am lebenslangen Lernen als einem Prozess des lebenslang reifer, erfahrener und gescheiter Werdens kann man nicht zweifeln. ${ }^{17}$ Die Theorieentwicklung tritt leider eindeutig hinter dem ökonomischen Diskurs zurück.

Die Verwendung des Konzepts informelles Lernen ${ }^{18}$, zunächst verstanden als ungeplant, beiläufig und von Lernenden nicht als Lernen intendiert, macht trotz nicht unerheblicher theoretischer und empirischer Schwierigkeiten und einer Vielzahl an unterschiedlichen Lesarten wirklich Sinn: Das informelle Lernen sollte zunehmend in der Arbeits- und Lebenswelt berücksichtigt werden, vor allem im Hinblick auf die hohe Jugendarbeitslosigkeit in Europa und die zunehmende Relevanz von Sozialisations- und Enkulturationsinstanzen, die sich ausserhalb der "klassischen” Instanzen von Familie und Schule etablieren: Freizeitwelten, Szenen, Subkulturen, virtuelle Welten. In der Regel wird als informelles Lernen bezeichnet, was als Lernen wahrgenommen und was ausserhalb etablierter Bildungsinstitutionen abläuft sowie auf irgendeine Weise mit „Outcomes” verbunden werden kann. Nach Dohmen stellt man sich mit der Beachtung dieses Phänomens „einer bisher vernachlässigten Grundform menschlichen Lernens für das lebenslange Lernen" - so der Untertitel zu seiner Expertise von 2001. Informelles Lernen hingegen ist die "natürliche Begleiterscheinung des täglichen Lebens. Anders als beim formalen und nicht formalen Lernen handelt es sich beim informellen Lernen nicht notwendigerweise um ein intentionales Lernen, weshalb es auch von Lernenden selbst unter Umständen gar nicht als

${ }^{17}$ Der hierzu geführte Diskurs ist primär einer der Beschäftigungsfähigkeit, der Employabilität. Dokumente zur Beschäftigungsinitiative, insbes. für die Jugend: Erste aktive Programme sind "Jugend in Bewegung", „European Alliance for Apprenticeships", EURES und Europass; Sonderprogramm MobiPro; vgl. auch <http://www.eu-bildungspolitik.de/ beschaeftigungsfaehigkeit_30.html> [15.10.2014].

18 Gemäß Philip Gonon (2002) stammt der Terminus von John Dewey. Die OECD befasst sich seit den 1990er Jahren mit dem Phänomen Informelles Lernen, um Bildungsgerechtigkeit, Innovationspotential und Wachstumsförderung zu fördern: Most research has focused on learning outcomes from formal education and training, instead of embracing all types of learning outcomes; allowing visibility and portability of such outcomes in the lifelong learning system, in the labour market or in the community. <http://www.oecd.org/edu/skills-beyondschool/recognitionofnon-formalandinformallearning-home.htm> [15.10.2014]. Die konsensfähige Definition der OECD ist: Informal learning is never organised, has no set objective in terms of learning outcomes and is never intentional from the learner's standpoint. Often it is referred to as learning by experience or just as experience. The idea is that the simple fact of existing constantly exposes the individual to learning situations, at work, at home or during leisure time for instance. This definition, with a few exceptions (see Werquin 2007) also meets with a fair degree of consensus. 
Erweiterung ihres Wissens und ihrer Fähigkeiten wahrgenommen wird."19 Mit der Idee des lebenslangen Lernens ist auf jeden Fall eine andere Perspektive entstanden, und zwar auf Lernen überhaupt. Statt Fixierung aufs Klassenzimmer: Ausweitung auf Lernorte. Dohmen (2001) argumentiert: „Es ist nicht entscheidend, ob ein solcher Begriff 'richtig' ist, sondern ob er fruchtbar und produktiv ist für ein notwendiges neues bildungspolitisches Sehen, Verstehen, Nachdenken und Handeln." (Dohmen 2001: 3)

\subsection{Lernort Szene: Subkulturelles und Musikszene}

Unsere FLL-Thematik wählt aus der Vielfalt von Szenen eine Musikszene aus, die sich von ihrer Soziosemiotik her deutlich von anderen Rockmusik-Szenen abhebt: die (Heavy) Metal-Szene. "Heavy” klammern wir ein, weil der Terminus Heavy Metal teils als Oberbegriff, teils als Subgenre verwendet wird. Wir heben mit unserer Schreibweise darauf ab, dass wir keine ideologisch und von der Bühnenperformance her extreme Richtung ins Auge fassen. Wir befassen uns nicht mit dem Musikstil des (Heavy) Metal, sondern mit der Musikszene (Heavy) Metal. In der Literatur zu popularen Kulturen und zur populären Musik wird „Genre (...) als übergeordneter Begriff verwendet, der auch 'aussermusikalische Elemente' enthält, während die mit dem Stilbegriff verbundene Ausdrücke eher das Musikalische betonen." (Elflein 2010: 32).

Auch wenn die Metal-Szene aktuell eine heterogene Altersstruktur bei Fans (und auch bei Bands!) aufweist, so können wir zum einen aufgrund ihrer Entstehungszusammenhänge von einer Jugendkulturszene sprechen, zum andern das „Bildungsprogramm” dieser Szene im Hinblick auf seine Anforderungen an jugendliche SzenegängerInnen untersuchen. ${ }^{20}$

Wir diskutieren hier nicht das Problem, inwieweit Rockmusik bzw. Metal auch Popmusik ist. Konsens besteht darin, dass Rockmusik sich „ästhe-

19 "Die von der OECD in den Leitlinien zu dieser Aktivität vorgelegte Definition nimmt andere Abgrenzungen vor: Formal learning refers to learning through a programme of instruction in an educational institution, adult training centre or workplace, which is generally recognised in a qualification or a certificate. Non-formal learning refers to learning through a programme but is not usually evalated and does not lead to a certification. Informal learning refers to learning resulting from daily work-related, familiy or leisure activities". (Stand der Anerkennung, S. 8)

${ }^{20}$ Da sich populäre Musik in westlichen Ländern nach dem II. Weltkrieg rasch und dynamisch in Bezug auf Musikschaffende und Fans entwickelte sind aktuell bestehende Musikszenen häufig mit "alten" Fans bestückt, die in einer Art Erinnerungskult Konzerte "damaliger" Bands besuchen bzw. sich in Radio-Wunschkonzerten Musikstücke "aus ihrer Jugend” wünschen und offenbar zuverlässig die entsprechenden Musikträger kaufen. 
tisch und soziologisch" definiert (Wicke, Ziegenrücker, Ziegenrücker 2007: 607) und in ihre multimodalen Kommunikaten einen ideologischen Code integriert. „Sie ist bestimmt durch die kollektive Identität von Texter, Komponist, Arrangeur und Interpret in der Rockgruppe. (...) In soziologischer Hinsicht ist Rockmusik aus der Lebensweise und Kultur Jugendlicher hervorgegangen und eingebettet in ein multimediales Umfeld, $\mathrm{zu}$ dem Druckgrafik und Fotografie (...), Film und Video (...), Literatur und Presse (...) gehören.” (ebd.: 607) „Mit dem britischen Beat war eine Musikpraxis entstanden, die zunächst ausserhalb des etablierten Medienzusammenhangs von den Jugendlichen selbst getragen und organisiert worden ist, von ihnen in ein Umfeld gestellt worden ist."

Musikszenen haben als Jugendkulturszenen zunächst subkulturelles Potential, ehe sie - verkürzt gesagt - von der Musikindustrie vereinnahmt werden. „We all want to change the world... - hiess es 1968 in dem BeatlesSong Revolution. Damit war ausgesprochen, was seit der bahnbrechenden Entwicklung des amerikanischen Rock'n'Roll ihren jugendlichen Fans als die Quintessenz der Popmusik gilt." (Wicke 2011: 7). Aktuelle RockmusikSzenen haben im Vergleich zu Schlager-, Volksmusik-, Techno-, House/RaveSzenen nach wie vor über "Love, Peace and Unity" hinausgehenden ideologischen Gehalt. Insofern ist Subkulturelles auch Teil des Bildungsprogramms, und wenn wir auf das Untersuchungsfeld „informelles Lernen in der Musikszene (Heavy) Metal" schauen, dann ist deutlich, dass (subjektiv und objektiv) Subkulturelles für Fans ein konstitutives Merkmal ihrer Szene ist. Subkulturelles ist hier durchaus Lerngegenstand, aber die Vergemeinschaftung ist in unseren Augen nicht zugleich und vor allem nicht vorab eine Subkultur. MetalRock setzt sich wie Musik generell von affirmativer populärer Musik auch dadurch ab, dass sie "echte” Musik im doppelten Sinn produziert: Die Metal-Musikschaffenden machen die Musik tatsächlich selbst, und ihr wird damit eine "Authentizität des Musizierens und im Gegensatz zu Popmusik" zugeschrieben (Wicke, Ziegenrücker, Ziegenrücker 2007: 607).

Für das Lernen in der Szene hat diese Zuschreibung hohe Relevanz, denn sie vermittelt damit den Kommunikaten Glaubwürdigkeit und hohes Orientierungspotential. In der Jugendkulturforschung wird das Phänomen Szene als Folge gesellschaftlicher Modernisierung bzw. der damit verbundenen Subjektivierungs-, Pluralisierungs- und Globalisierungs- und insbesondere der Individualisierungsprozesse gesehen: „Sie verändern auch die klassischen Gesellungsformen", wie etwa Nachbarschaft, Vereine, Parteien (Hitzler, Niederbacher (2010: 11ff.). Diese Entwicklung ist ambivalent: „Einerseits wird das Individuum aus überkommenen Bindungen freigesetzt, 
wodurch es mehr Entscheidungschancen und Lebenspositionen erlangt. Andererseits verliert es nicht nur (...) gemeinschaftliche, sondern zusehends auch bislang gesellschaftlich 'garantierte' Verlässlichkeit (...)." (Hitzler, Niederbacher 2010: 12) Aus dieser Ambivalenz und auch aus den freizeitlichen Wahlmöglichkeiten und - notwendigkeiten heraus steigen Anforderungen an Jugendliche: „Einerseits gilt es, aus der (Über)Fülle des Wähl-, Nutz- und Machbaren eine bestimmte Kombination von Konsum- und Erlebnisoptionen für sich als wünschbar zu setzen - wobei diese Präferenz selten 'einsam' geschieht, sondern deutlich mit der Partizipation an Lebensstilformationen und eben an 'Szenen' korreliert." Bildung - im weiten Sinne der Entwicklung und Aneignung lebenspraktisch relevanter Kompetenzen - erwerben immer mehr Jugendliche heutzutage in diesen 'ihren', gegenüber anderen Lebensbereichen relativ autonomen freizeitlichen Sozialräumen (Hitzler, Niederbacher 2010: 13 f.).

Jugendliche entwickeln und erwerben gerade in Szenen soziosemiotische Kompetenzen, die für ihren Alltag, ihre Position in den Gleichaltrigengruppen und für ihre Partizipation am Szene- und Alltagsleben entscheidend sind. Die Frage ist nicht, ob sie sich die Codes bewusst aneignen, die Frage ist, ob sie die Codes als Codes lesen lernen und sie in einen symbolischen Rahmen stellen und in ihnen Sublimierungsprozesse problematischer Realitäten sehen. Im Hinblick auf Kompetenzerwerb geht es bei der Wahrnehmung der multimodalen Kommunikate darum, Kohärenz herzustellen und die richtigen ästhetischen Entscheidungen zu treffen: Sind Änderungen der Kommunikate einer Band zulässig? Oder weisen die Änderungen auf Vereinnahmung durch die Musikindustrie hin? Diese Deutungen scheinen dann besonders wichtig zu sein, wenn sich die Szene ausdrücklich nicht in den Mainstream integrieren lassen will, wenn sie ihr Protestpotential erhalten will, wenn sie für sich und "ausserhalb" bleiben will. Die Gefahr des Einverleibtwerdens ist durchaus realistisch.

Populäre Musikrichtungen haben als einziges gemeinsames Merkmal die mehr oder weniger ausgeprägte strukturelle und funktionale Prägung durch die Kulturindustrie. Mit "mehr oder weniger” weisen wir darauf hin, dass sich trotz der Tatsache, dass Kulturindustrie ein integraler - evtl. auch konstitutiver - Teil der westlichen kapitalistischen Gesellschaftssysteme ist, immer wieder Musikszenen, Subkulturen, Szenen der vollständigen kulturindustriellen Prägung entziehen, um allerdings innert Kürze dann doch vereinnahmt zu werden, sobald eben Dissemination erfolgt. „Die musikalischen Guerillakämpfer der diversen Subkulturen haben die Wertschöpfungskette lediglich fantasievoller bedient, diese abzuschaffen oder auch nur das Diktat von Chartpositionen, Einschaltquoten und Verkaufszahlen zu 
relativieren, vermochten sie nicht. Im Gegenteil, der Gestus der Rebellion und die Attitüde des Revolutionärs erwiesen sich lange Zeit als eines der wirkungsvollsten Marketingkonzepte der Branche." (Wicke 2011: 11).

Es ist daher leicht einzusehen, dass sich Musikszenen mit subkulturellem Gehalt und einer Anti-Mainstream-Haltung nicht nur aus einer Protesthaltung heraus als unabhängig darzustellen versuchen: auch aus semiotischästhetischen Gründen müssen sie sich als „unvereinnahmt” und „unkommerziell" darstellen.

Auch die Ausdifferenzierung in Metal-Subgenres, die soziosemiotische Kompetenzen fordert, scheint weniger der musikalisch-mentalitären Vielfalt geschuldet als vielmehr einschlägiger Gesetzmässigkeiten der Kulturindustrien. Nachdem zu einen in den 1980er Jahren die Einführung neuer Technologie zu Umsatzeinbrüchen beim Verkauf von Tonträgern geführt hatte und zum andern der Pop-Mainstream endgültig in Programmformaten der Medien keine "musikalische Synthese aus unabhängig voneinander entwickelten Musikformen” mehr darstellte, sondern „die disparate Vielfalt (...) nach dem Supermarktprinzip in einen vereinheitlichenden medialen Kontext" (Wicke 2011: 95) stellte, änderte die Kulturindustrie ihr Marktkonzept: Statt Massenproduktion herausragender Stile, Stars, Bands werden jetzt Minderheiten bedient, das heisst Minderheiten geschaffen. „Fortan setzte sich ein zielgruppenorientierters Konzept durch, das sich im Kern bis heute erhalten hat. Seither wird der Markt mit neuen Produkten und Produktkategorien (Musikstilen), die auf genau umrissene Zielgruppen stellen, förmlich überschüttet" (Wicke 2011: 97 f.). ${ }^{21}$

\subsection{Ausgewählte Szene: (Heavy) Metal}

An keiner Musikszene scheiden sich die Geister derart drastisch. Studierende der Bergischen Universität Wuppertal reagieren bei Befragungen 2014 zur Einschätzung des Metal mit geradezu aggressiven Formulierungen (obwohl es unter anderem um eindeutig gesellschaftskritische Lyrics ging), Frau Gore und ihre Vereinigung Parents Music Resource Center (PMRC) führte 1985 einen Kreuzzug gegen „Pop, Rock und Heavy Metal” (Wehrli 2012: 63), bei dem es vor allem darum ging, Heavy Metal „für soziale Devianz,

${ }^{21}$ „Die Kleinteiligkeit der Zielgruppen wird mehr als wettgemacht durch die Summierung der Gewinnzyklen innerhalb eines gegebenen Zeitraums. Das Gesetz der Ökonomie der grossen Zahl (...) - die Masse es also macht (economics of scale) -, wurde von einer Strategie abgelöst, die auf Effizienz und Umlaufgeschwindigkeit des investierten Kapitals setzt (economics of scope)." (Wicke 2011: 98) 
Jugendkriminalität und Drogensucht" (ebd.: 67) unmittelbar verantwortlich zu machen. Eine US-Metalforscherin namens Kirsten Dyck sieht im Jahr 2012 im Gebrauch nordisch-heidnischer Symbolik nationalsozialistischfaschistoide Haltungen. Und sogar im Handbuch der populären Musik (Wicke, Ziegenrücker, Ziegenrücker 2007: 435) steht beim dem Stichwort Metal Punk: „(...) Das sozialkritische Element, das dem britischen Punk Rock noch eigen war, ist hier durch einen ungebrochenen Kult der Gewalt um ihrer selbst willen ersetzt." Damit nimmt auch das Handbuch eine Art semiotische Enteignung vor; es verweigert eine symbolischen Semiose zugunsten einer indexikalischen. Bei Reaktionen auf Metal im Alltag, bei Feldforschungen, in wissenschaftlicher Literatur finden sich größtenteils indexikalisch fundierte Semiosen, so dass man geradezu von einer "dual-use-Problematik" sprechen muss, von einem Zweifachnutzen der Metal-Kommunikate. Studierende, die im SS 2010 an Feldforschungen in Wuppertaler und Solinger Metalszene-Lokalen teilnahmen, kamen völlig irritiert in die nächste Seminarsitzung zurück: „Die [die Metalheads, die sie getroffen und interviewt haben] sind ja sooo lieb." Als ob Menschen, die sich sozialkritisch geben, das verkörpern, wogegen sie protestieren.

Diese Dialektik macht diese Musikszene ausserordentlich interessant und ergiebig für die Auseinandersetzung mit ihrem Bildungsprogramm, ihrer Soziosemiotik und Multimodalität.

Wicke, Ziegenrücker und Ziegenrücker (2007: 312) nennen die Stilrichtung, die wir (Heavy) Metal nennen, Heavy Metal Rock und charakterisieren sie als „eine Spielweise der Rockmusik, die musikalisch weitgehend mit dem Hard Rock früherer Jahre identisch ist, sich von diesem allenfalls durch grössere Lautstärken und ein dank moderner Aufnahmetechnik schwergewichtigeres, von verzerrten E-Gitarren dominiertes Klangbild unterscheidet." Sie nennen sie "die radikalste Ausprägung der Rockmusik”. „In ihr verkörpert sich eine soziale Ventilfunktion, die ihre Basis in den unterpriveligierten Teilen der Jugend besitzt und mit wachsenden sozialen Spannungen, ökonomischen Krisenerscheinungen und ansteigender Jugendarbeitslosigkeit deutlich an Aggressivität zugenommen hat. War der Hard Rock der end1960er Jahre noch durch einen provokanten Primitivismus gekennzeichnet, der soziales Konfliktpotential und Frustrationen in Lautstärkeorgien abreagierte, so steigerte sich das zehn Jahre später in einen aggressiven Kult der Gewalt mit noch höherem Lautstärkepegel, einen makabren Okkultismus oder einen brutalen Männlichkeitsfetischismus." Da Männlichkeitsfetischismus in allen Arten von Szenen (und nicht nur da) allüberall beobachtet werden kann, muss man sich fragen, warum es gerade der Me- 
tal-Szene zum Vorwurf gereicht. Männer der Skater-Szene ignorieren ja beharrlich die Kunstfertigkeit weiblicher Menschen, die Skateboard fahren.

Vielleicht liegt die negative Einschätzung an der spezifischen Kombination der Codes? Der Transgression (Kahn-Harris 2007 über Extreme Metal) in allen Modalitäten?

In Heavy Metal begegnet uns ein breites und komplex ineinander verschachteltes Spektrum an Codes: Metal realisiert wie jede Musikszene nicht „nur" Musik, sondern vielfältige visualisierte Äußerungen und Handlungen sowie Verbales innerhalb der Musik wie auch als expliziter poetischer Text.

\subsection{Szene-Lerngegenstand Multimodalität und Soziosemiotik}

Uns scheint, dass in Musikszenen bzw. in Gleichaltrigengruppen durch informelles Lernen soziosemiotische Kompetenzen vor allem für multimodale Kommunikate erworben werden, die ausserhalb solcher Szenen nicht erworben werden können. Auch die Strategien für den Auf- und Ausbau der soziosemiotischen Kompetenzen werden in und durch die Musikszene erworben. Das gilt umso mehr als "praktisch jede Szene mit zunehmendem Entwicklungsstadium zu einem hochgradig differenzierten Konglomerat von Teil- und Subszenen wird - oder neue Szenen generiert (vgl. exemplarisch die Entstehung von Hardcore aus dem Punk)." (Hitzler, Niederbacher 2010: 191).

Zentraler, genuiner Lerngegenstand ist aus kultursemiotischer Sicht die Soziosemiotik multimodaler Kommunikate. Das intuitive Verstehen der Zeichenhaftigkeit des Sprachlich-Kommunikativen in Szenen, zu denen man gehört, und das Wissen, dass verbale und non-verbale Äußerungen in ihrer Bedeutung immer "Sagen” und "Meinen” umfassen, ist die Grundvoraussetzung dafür, dass man sich einer Szene zugehörig fühlt und dass man als zu der Szene zugehörige Person betrachtet wird. Wenn die Mitglieder einer Szene nicht einvernehmlich ihre materiale Kultur und ihre Codes konstituieren oder auch nur rezipieren, dann gibt's keine Szene, keine Vergemeinschaftung. Wahrscheinlich haben alle Szenen - ob Antifa oder Hausbesetzer oder Veganer - einen Code für Ästhetik; bei Musikszenen ist der ästhetische Code eine notwendige Bedingung. „Es ist bereits deutlich geworden, dass Szenen ihre Kohäsion aus ästhetisch-stilistischen Gemeinsamkeiten im Hinblick auf einen bestimmten thematischen Fokus beziehen. Diese Gemeinsamkeiten müssen kommunikativ und interaktiv 'in Szene gesetzt' werden, weil sie ansonsten als für die Beteiligten 'unsichtbar' deklariert werden müssen (...)." (Hitzler, Niederbacher 2010: 27). 
Jede Szene ist für die SzenegängerInnen eine semiotische, ästhetische und eine soziale "Schulung”. Der Lerngegenstand - das "Bildungsprogramm" - ist umfassend. Im Zentrum stehen bei Musikszenen die multimodalen Kommunikate, die es sowohl im Zeichensystem der Szene als auch im umfassenden der die Szene umfassenden Welt zu verstehen gilt. Das soziosemiotische System der Metal-Welt ist nur auf dem Hintergrund der Zeichensysteme der umfassenden Welt zu verstehen. Es muss also eine Kontextsensitivität für Zeichen und ihren Gebrauch entwickelt werden. Es geht dabei auch um die Erfahrung, dass es sehr viele Ressourcen von Zeichen gibt, aus denen die szenespezifischen ausgewählt, dekontextualisiert und neu kontextualisiert werden. Wir verwenden das Konzept Bricolage, um diese Prozesse zu beschreiben.

Beim musikalischen Code werden von Fans Abweichungen und Konstanten registriert und bewertet: „Ein Musikstil wie Heavy Metal hat damit eine bestimmte, quasi normative Klangfarbe, aber keinen normativen Klang. Nichtsdestotrotz ist die Klangfarbe ein Bereich des Traditionsstroms, der Veränderungen unterworfen ist." (Elflein 2010: 72). Dabei geschieht immer ein horizontales Vergleichen - zwischen Band zum Beispiel - und ein vertikales, ein chronologisches.

Dasselbe gilt für sämtliche visuellen Codes in all ihren verschiedenen Erscheinungsformen: Von Symbolen beim Coverartwork über Kleidung und Haartracht zur Aktivität einer Band auf der Bühne. Betrachten wir allein die Intertextualität von Metal-Flyern wird sofort plausibel, dass semiotische Kompetenz erworben wird. Ist diese Kompetenz auf Glokales angelegt?

Dasselbe gilt für verbale Zeichen und Texte.

Alle Codes brauchen Wahrnehmungs- und Interpretationsrahmen für eigene und fremde, für alte und neue "Codes", die auf einer lokalen und auf einer globalen Ebene angesiedelt sind. Um dieses Sowohl-als-auch ausdrükken zu können, spricht man Glokalität. Neben der Kompetenz, die Kommunikate $\mathrm{zu}$ verstehen, entwickelt sich noch eine interaktiv-kommunikative Kompetenz. Mit der Feststellung "Szenen sind kommunikative und interaktive Teilzeit-Gesellungsformen" kondensieren Hitzler, Niederbacher (2010) die Beschreibungen der soziologische und pädagogischen Jugendkulturforschung: Die Existenz von Szenen ist nicht nur ", an die ständige kommunikative Vergewisserung" gebunden, „sondern vielmehr an die ständige kommunikative Erzeugung gemeinsamer Interessen seitens der Szenegänger: Im sinnlich erfassbaren Gebrauch szenetypischer Symbole, Zeichen, Rituale inszenieren diese ihre eigene Zugehörigkeit und konstituieren tatsächlich zugleich, sozusagen 'beiläufig', die Szene. Vor allem in diesem Sinne lässt sich eine Szene mithin als Netzwerk von Personen verstehen, die bestimmte 
materiale und/oder mentale Formen der kollektiven (Selbst-)Stilisierung teilen und diese Gemeinsamkeiten kommunikativ stabilisieren, modifizieren oder transformieren" (Hitzler, Niederbacher 2010: 17).

Es liegt ein Kreislauf vor, der die Attraktivität von Szenen als Sozialisations- und Enkulturationsinstanzen noch erhöht: Um Mitglied einer frei gewählten Szene zu werden, kann man am Anfang lediglich die Faszination für den Szenefokus mit andern teilen. Damit aber die Szene weiterbesteht, stabil ist und den Zuspruch bringt, den man sich verspricht, muss man zum Erhalt der Szene beitragen, indem man die für die Szene richtigen Aktivitäten erlernt und realisiert und wenn erforderlich modifiziert. Damit werden Reflexion und Verantwortung erlernt und in einer aufgeschlossenen Szene wie der Metal-Szene auch thematisiert. Es scheint sich dabei die Altersmischung der Metal-Szene positiv auszuwirken: Die alten Metal-Heads stellen ihr Wissen zur Disposition, und sie lehren die jungen Metal-Heads über die soziosemiotischen Inhalte hinaus auch die moralischen Massstäbe.

Aus dieser Bildungsperspektive heraus und aufgrund unserer kultursemiotisch-linguistischen Ausrichtung und unserem Fokus auf multimodale Kommunikate gehen wir von folgenden Parametern aus: I History \& Story, II Cultures \& Values, III Sounds, IV Lyrics, V Looks \& Gestures, VI Symbols, VII Marketing, VIII Glokalität, die in FLL-Publikationen und im Forschungsprojekt "Soziosemiotik des europäischen Heavy Metal“ beschrieben werden.

\section{LITERATURVERZEICHNIS}

Aebli, H., 1980. Denken: Das Ordnen des Tuns. Band I: Kognitive Aspekte der Handlungstheorie. Stuttgart: Klett-Cotta.

Aebli, H., 1981. Denken: Das Ordnen des Tuns. Band II: Denkprozesse. Stuttgart: Klett-Cotta.

Aebli, H., 1981 [1976]. Grundformen des Lehrens. Eine Allgemeine Didaktik und kognitionspsychologischer Grundlage. Stuttgart: Klett-Cotta.

Aepkers, M., Liebig, S., 2002. Entdeckendes, Forschendes und Genetisches Lernen. Baltmannsweiler: Schneider Verlag Hohengehren.

Alkemeyer, T. 2003. Semiotische Aspekte der Soziologie: Soziosemiotik. In: Posner, v. R., Robering, K., Sebeok, Th.A. (Hrsg.). Semiotik, Semiotics. Ein Handbuch zu den Zeichentheoretischen Grundlagen von Natur und Kultur. Berlin: de Gruyter, 2757-2846.

Baacke, D., 1968. Beat - die sprachlose Opposition. München: Juventa.

Baacke, D., 1972. Jugend und Subkultur. München: Juventa.

Baacke, D., 1976. Die 13- bis 18jährigen. Einführung in Probleme des Jugendalters. München: Urban \& Schwarzenberg.

Baacke, D., 1987. Jugend und Jugendkulturen. Darstellung und Deutung. Weinheim: Juventa.

Bartosch, R. (Hrsg.) 2011. Heavy Metal Studies. Bd 1: Lyrics und Intertextualität. Oberhausen: Verlag Nicole Schmenk. 
Bundesministerium für Bildung und Forschung (BMBF), 2008. Stand der Anerkennung non-formalen und informellen Lernens in Deutschland. Bonn, Berlin.

Chaker, S., 2011. Götter des Gemetzels. Mediale Inszenierungen von Tod und Gewalt im Death Metal. In: Bartosch, R. (Hrsg.). Heavy Metal Studies. Bd 1. Lyrics und Intertextualität. Oberhausen: Verlag Nicole Schmenk, 11-24.

Diederichsen, D. 2014. Über Popmusik. Köln: Kiepenheuer \& Witsch.

Diederichsen, D., Hebdige, D., Marx, O.-D. 1983. SCHOCKER. Stile und Medien der Subkultur. Reinbek bei Hamburg: Rowolth.

Dohmen, G., 1996. Das Lebenslange Lernen. Leitlinien einer modernen Bildungspolitik. Bonn: Bundesministerium für Bildung, Wissenschaft, Forschung und Technologie.

Dohmen, G., 2001. Das informelle Lernen. Die internationale Erschließung einer bisher vernachlässigten Grundform menschlichen Lernens für das lebenslange Lernen aller. Bonn: Bundesministerium für Bildung und Forschung.

Dunn, S., 2005. Metal. A Headbanger's Journey. DVD. Constantin Film.

Dunn, S., 2008. Global Metal. DVD. Universal.

Düx, W. (Hrsg.), 2009. Kompetenzerwerb im freiwilligen Engagement. Eine empirische Studie zum informellen Lernen im Jugendalter. Wiesbaden: VS Verlag.

Dyck, K., 2012. „The Blood of my Ancestors” - Nostalgia in White-Supremacist Hate Rock. In: Falke, S.R., Wisotzki, K. (Hrsg.). Böse Macht Musik. Zur Ästhetik des Bösen in der Musik. Bielefeld: transcript Verlag, 131-139.

Elflein, D., 2010. Schwermetallanalysen. Die musikalische Sprache des Heavy Metal. Bielefeld: transcript Verlag.

Falke, S.R., Wisotzki, K., 2012. Böse Macht Musik. Zur Ästhetik des Bösen in der Musik. Bielefeld: transcript Verlag.

Farin, K., Neubauer, H., 2001. Artificial Tribes. Jugendliche Stammeskulturen in Deutschland. Berlin: Archiv der Jugendkulturen, Verlag Thomas Tilsner.

Ferchhoff, W., 1999. Jugend an der Wende vom 20. zum 21. Jahrhundert. Opladen: Leske + Budrich.

Ferchhoff, W., 2010. Jugend und Jugendkulturen im 21. Jahrhundert. Wiesbaden: VS Verlag.

Ferchhoff, W., Neubauer, G., 1997. Patchwork-Jugend. Eine Einführung in postmoderne Sichtweisen. Opladen: Leske + Budrich.

Fishman, J.A., 1975. Soziologie der Sprache. Eine interdisziplinäre sozialwissenschaftliche Betrachtung der Sprache in der Gesellschaft. München: hueber.

Gonon, P. 2002. Informelles Lernen - ein kurzer historischer Abriss von John Dewey zur heutigen Weiterbildung. In: Dehnbostel, P., Gonon, P. (Hrsg.). Informelles Lernen - eine Herausforderung für die berufliche Weiterbildung, Bielefeld: Bertelsmann, 13-22.

Harring, M., Rohlfs, C., Palentien, Ch., 2007. Perspektiven der Bildung. Kinder und Jugendliche in formellen, nicht-formellen und informellen Bildungsprozessen. Wiesbaden: VS Verlag.

Hecken, T., 2009. POP. Geschichte eines Konzepts 1955-2009. Bielefeld: transcript Verlag.

Heesch, F., 2011. Thor im Metal und im Fantasy-Roman. Zur "Asgard-Saga" von Manowar und Wolfgang Hohlbein. In: Bartosch, R. (Hrsg.). Heavy Metal Studies. Bd. 1. Lyrics und Intertextualität. Oberhausen: Verlag Nicole Schmenk, 64-88.

Hitzler, R., Bucher, T., Niederbacher, A. 2000. Jugendszenen (in NRW). Uber juvenile Kultur(en) unter den Bedingungen der Spätmoderne. Düsseldorf (Expertise im Auftrag des Ministeriums für Frauen, Jugend, Familie und Gesundheit des Landes NordrheinWestfalen. 7. Kinder und Jugendbericht der Landesregierung NRW). 
Hitzler, R., Bucher, T., Niederbacher, A., 2001. Leben in Szenen. Formen jugendlicher Vergemeinschaftung heute. Opladen: Leske + Budrich.

Hitzler, R., Niederbacher, A., 2010. Leben in Szenen. Formen juveniler Vergemeinschaftung heute 3. Vollständig überarbeitete Auflage. Wiesbaden: VS Verlag.

Hitzler, R., Pfadenhauer, M. 2005. Unsichtbare Bildungsprogramme? Zur Entwicklung und Aneignung praxisrelevanter Kompetenzen in Jugendszenen. Düsseldorf. (Expertise zum 8. Kinder- und Jugendbericht der Landesregierung NRW).

Huber, L., Hellmer, J., Schneider, F., 2009. Forschendes Lernen im Studium. Aktuelle Konzepte und Erfahrungen. Bielefeld: UniversitätsVerlag Webler.

Hügel, H.-O., 2003. Einführung. In: Handbuch Populäre Kultur. Begriffe, Theorien und Diskussionen. Hrsg. H.-O. Hügel. Stuttgart: Metzler, 1-22.

Hügel, H.-O. (Hrsg.)., 2003. Handbuch Populäre Kultur. Begriffe, Theorien und Diskussionen. Stuttgart: Metzler.

Hügel, H.-O. (Hrsg.) 2007. Lob des Mainstreams. Zu Begriff und Geschichte von Unterhaltung und Populärer Kultur. Köln: Herbert von Halem Verlag.

Hurrelmann, K., 2004. Lebensphase Jugend. Eine Einführung in die sozialwissenschaftliche Jugendforschung. Weinheim: Juventa 7., vollständig überarbeitete Auflage.

Hurrelmann, K., Quenzel, G., 2012. (19851). Lebensphase Jugend. Eine Einführung in die sozialwissenschaftliche Jugendforschung. Weinheim: Beltz Juventa 11., vollständig überarbeitete Auflage.

Kahn-Harris, K., 2007. Extreme Metal. Music and Culture on the Edge. Oxford: Berg.

Kommission der Europäischen Gemeinschaften. 2000. Memorandum uber lebenslanges Lernen. Brüssel. KOM (2000) 1832.

Kommission der Europaïschen Gemeinschaften. 2001. Einen europaïschen Raum des lebenslangen Lernens schaffen. Brüssel. KOM (2001) 678.

Krüger H.-H., Grunert, C., 2002. Handbuch Kindheits- und Jugendforschung. Opladen: Leske + Budrich.

Lang, E., Werlen, L., 2013. Multimediale Variation und informelles Lernen: Soziosemiotik der Heavy-Metal-Szene aus soziolinguistischer und Kulturmanagement-Perspektive. In: Albert, G., Franz, J. (Hrsg.) Zeichen und Stil. Der Mehrwert der Variation. Festschrift für Beate Henn-Memmesheimer. Frankfurt am Main: Peter Lang: 159-177.

Lang, E., Werlen, L. (i.E.). Soziosemiotik und Multimodalität der (Heavy) Metal-Szene. Hohengehren: Schneider Verlag Hohengehren

Lotman, J.M., 1981. Kunst als Sprache. Untersuchungen zum Zeichencharakter von Literatur und Kunst. Leipzig: Verlag Philipp Reclam jun.

Molzberger, G,. 2007. Rahmungen informellen Lernens. Zur Erschließung neuer Lern- und Weiterbildungsperspektiven. Wiesbaden: VS Verlag.

Morris, Ch.W., 1977. Pragmatische Semiotik und Handlungstheorie. Frankfurt am Main: Suhrkamp.

Morris, Ch.W., 1979. Grundlagen der Zeichentheorie. Ästhetik und Zeichentheorie. Frankfurt am Main: Ullstein.

Nohr, R.F., Schwaab, H., 2012. Metal Matters. Heavy Metal als Kultur und Welt. Münster: Lit Verlag 2., unveränderte Auflage.

Noltteernsting, E., 2001. „Wir hören absolute Kieferbrecher und Schädelspalter”. Heavy Metal. In: Farin, K., Neubauer H. (Hrsg.). Artificial Tribes. Jugendliche Stammeskulturen in Deutschland. Berlin: Archiv der Jugendkulturen,Verlag Thomas Tilsner, 150-173.

Nünning, A., Nünning, V., 2008. Einführung in die Kulturwissenschaften. Theoretische Grundlagen - Ansätze - Perspektiven. Stuttgart: Metzler. 
Overwien, B., 2007. Informelles Lernen - zum Stand der internationalen Diskussion. In: Rauschenbach T., Düx W., Sass E. (Hrsg.). Informelles Lernen im Jugendalter. Vernachlässigte Dimensionen der Bildungsdebatte. Weinheim: Juventa, 35-62.

Posner, R., 2008. Kultursemiotik. In: Nünning A., Nünning V. (Hrsg.). Einführung in die Kulturwissenschaften. Theoretische Grundlagen - Ansätze - Perspektiven. Stuttgart: Metzler, 39-72.

Rauschenbach, T., Düx, W., Sass, E. 2007. Informelles Lernen im Jugendalter. Vernachlässigte Dimensionen der Bildungsdebatte. Weinheim: Juventa.

Richard, B., Grünwald, J., 2012. Verführer und Zerstörer - mediale Bilder archaischer Männlichkeit im Black Metal. In: Nohr R.F., Schwaab H. (Hrsg.). Metal Matters. Heavy Metal als Kultur und Welt. Münster: Lit Verlag 2., unveränderte Auflage, 43-53.

Roccor, B., 1998. Heavy Metal. Die Bands. Die Fans. Die Gegner. München: Beck.

Ruile, A.M., 2010. Lernen in Jugendszenen. Ein Ausweg aus sozialer Ungleichheit im Bildungssystem? Marburg: Tectum.

Schäffer, B., 1996. Die Band. Stil und ästhetische Praxis im Jugendalter. Opladen: Leske + Budrich.

Schröder, A., 2007. Cliquen und Peers als Lernort im Jugendalter. In: Rauschenbach T., Düx W., Sass E. (Hrsg.). Informelles Lernen im Jugendalter. Vernachlässigte Dimensionen der Bildungsdebatte. Weinheim: Juventa, 173-202.

Spitzmüller, J. 2013. Graphische Variation als soziale Praxis. Eine soziolinguistische Theorie skripturaler «Sichtbarkeit». Berlin: De Gruyter.

Steiner, G., 2007. Der Kick zum effizienten Lernen. Erfolgreich und nachhaltig ausbilden dank lernpsychologischer Kompetenz - vermittelt an 30 Beispielen. Bern: h.e.p. verlag ag.

Stöckl, H., 2004. Die Sprache im Bild - das Bild in der Sprache. Zur Verknüpfung von Sprache und Bild im massenmedialen Text. Konzepte, Theorien, Analysemethoden. Berlin: de Gruyter.

Thole, W., 2002. Jugend, Freizeit, Medien und Kultur. In: Krüger H.-H., Grunert C. (Hrsg.). Handbuch Kindheits- und Jugendforschung. Opladen: Leske + Budrich, 653-684.

Warneken, B.J., 2006. Die Ethnographie popularer Kulturen. Eine Einführung. Wien: Böhlau.

Wehrli, R., 2012. Verteufelter Heavy Metal. Skandale und Zensur in der neueren Musikgeschichte. Münster: Telos Verlag. Korrigiertes und ergänztes Update.

Weinstein, D., 1991. Heavy Metal - A Cultural Sociology. New York: Lexington.

Wicke, P., 2011. Rock und Pop. Von Elvis Presley bis Lady Gaga. München: Verlag C.H. Beck.

Wicke, P., Ziegenrücker, W., Ziegenrücker, K.-E., 2007. Handbuch der populären Musik. Geschichte, Stile, Praxis, Industrie. Mainz: Schott Music GmbH \& Co. KG. Erweiterte Neuausgabe. 